\title{
Acute myeloid leukemia with CEBPA somatic mutations
}

INSERM

\section{Source}

INSERM. (1999). Orphanet: an online rare disease and orphan drug data base. Acute myeloid leukemia with CEBPA somatic mutations. ORPHA:319480

Acute myeloid leukemia with CEBPA somatic mutations is a subtype of acute myeloid leukemia with recurrent genetic abnormalities, characterized by clonal proliferation of myeloid blasts harboring somatic mutations of the CEBPA gene in the bone marrow, blood and, rarely, other tissues. It can present with anemia, thrombocytopenia, and other nonspecific symptoms related to ineffective hematopoesis (fatigue, bleeding and bruising, recurrent infections, bone pain) and/or extramedullary site involvement (ging ivitis, splenomegaly). 